\title{
Bilateração e Geometria de Distâncias
}

\author{
Germano Abud, \\ Faculdade de Matemática, FAMAT, UFU, 38408-100, Uberlândia, MG. \\ E-mail: germano@famat.ufu.br \\ Jorge Alencar, Carlile Lavor, \\ Instituto de Matemática, Estatística e Computação Científica, DMA, IMECC, UNICAMP, \\ 13083-859, Campinas, SP. \\ E-mail: jorge.fa.lima@gmail.com, clavor@ime.unicamp.br
}

Resumo: Em [3], os autores estabelecem uma "dualidade" entre o DGP (Problema de Geometria de Distâncias) e o EDMCP (Problema de Completamento de Matrizes de Distâncias Euclidianas). Neste trabalho, mostraremos como o teorema sobre o número de soluções (em $[1,5])$ pode ser utilizado em conjunto com a bilateração, para o cálculo de certas distâncias desconhecidas. O teorema sobre número de soluções é válido para instâncias de um ${ }^{K} D M D G P$ $(K \geq 1)$, mas trataremos apenas do caso $K=2$, para o qual faz sentido aplicar o método de bilateração.

Palavras-chave: Geometria de distâncias euclidianas, completamento de matrizes, bilateração

\section{Introdução}

Menger [6] caracterizou vários conceitos geométricos (congruência, convexidade, etc.) em termos de distâncias. Os resultados de Menger e algumas complementações apresentadas posteriormente por Blumenthal [2] deram origem a um ramo de estudo denominado Geometria de Distâncias, cujo principal problema pode ser assim enunciado:

Problema de Geometria de Distâncias (DGP ${ }^{1}$ ): Dado um natural $K>0$ e um grafo simples, ponderado, não direcionado, $G=(V, E, d)$, cujas arestas são ponderadas por uma função $d: E \rightarrow \mathbb{R}_{+}$, determine se existe uma função $x: V \rightarrow \mathbb{R}^{K}$ tal que

$$
\forall\{u, v\} \in E,\|x(u)-x(v)\|=d(\{u, v\}) ;
$$

onde $\|\cdot\|$ indica a norma euclidiana. A função $x$ em (1) é dita uma realização válida de $G$ em $\mathbb{R}^{K}$.

Duas realizações $x$ e $y$ de um grafo $G$ são ditas congruentes, se

$$
\|x(u)-x(v)\|=\|y(u)-y(v)\|, \forall u, v \in V .
$$

Em outras palavras, elas são congruentes se uma pode ser obtida a partir da outra, por uma composição de rotações, reflexões e translações.

O interesse no DGP está relacionado à riqueza de suas aplicações (conformação molecular, redes de sensores sem fio, visualização de dados e robótica, entre outros), bem como na beleza da teoria matemática envolvida [4]. Uma subclasse importante de problemas é a seguinte:

$K$ - Problema Discretizável de Geometria de Distâncias Moleculares $\left({ }^{K} \mathbf{D M D G P}^{2}\right)$ :

Dado um inteiro positivo $K$, um grafo simples, ponderado, não-direcionado, $G=$

\footnotetext{
${ }^{1}$ Do inglês: Distance Geometry Problem

${ }^{2}$ Do inglês: Discretizable Molecular Distance Geometry Problem
} 
$(V, E, d)$, onde $d: E \rightarrow \mathbb{R}_{+}$, uma ordem $<$em $V$ tal que $\{u, v\} \in E$, para cada $v>K$ e $v-K \leq u \leq v-1$ (conhecemos todas as distâncias entre quaisquer $K+1$ vértices consecutivos), e uma realização parcial $\bar{x}:\{1, \ldots, K\} \rightarrow \mathbb{R}^{K}$, desejamos saber se existe uma realização $x: V \rightarrow \mathbb{R}^{K}$, tal que

$$
\forall\{u, v\} \in E \quad\|x(u)-x(v)\|=d(\{u, v\}),
$$

e tal que $x(v)=\bar{x}(v)$ para cada $v \in\{1, \ldots, K\}$.

Seja $X$ o conjunto de todas as soluções (realizações válidas) de um ${ }^{K}$ DMDGP. De [4], sabemos que se existe uma ordem sobre o conjunto de vértices $V$, tal que cada vértice $v$, com $v>K$, tem exatamente $K$ predecessores adjacentes, então (com probabilidade 1) temos $|X|=2^{n-K}$. Se existem pelo menos $K$ predecessores adjacentes então $|X| \leq 2^{n-K}$.

No presente trabalho, trataremos da classe de problemas ${ }^{2}$ DMDGP $(K=2)$. Vamos particionar $E$ em dois conjuntos $E_{D}$ e $E_{P}$, tais que $E_{D}=\{\{i, j\} \in E:|i-j| \leq 3\}$ e $E_{P}=E \backslash E_{D}$. Uma aresta em $E_{P}$ é dita aresta de poda e uma aresta em $E_{D}$ é dita aresta de discretização.

De acordo com as observações acima, uma maneira natural de explorar a situação é efetuar uma busca em uma árvore, que "ramifica" no vértice $v$, ao computar as possibilidades $x_{v}^{0}$ e $x_{v}^{1}$ e, quando possível, "poda" um ramo $x_{v}^{\ell}, \ell \in\{0,1\}$, se existe uma aresta de poda $\{u, v\}$, cujo peso $d_{u v}$ é incompatível com a posição $x_{v}^{\ell}$. Este método é conhecido como Branch-and-Prune (BP) $[4]$.

A cada passo do BP, podemos localizar o $i$-ésimo átomo em duas posições $x_{i}$ e $x_{i}^{\prime}$. Entretanto, alguma ou ambas as posições podem ser infactíveis, com respeito a certas restrições sobre as distâncias. A busca se ramifica em todas as posições atômicas que são factíveis com relação a todas as restrições e, se uma posição não é factível, o escopo da busca é podado. Uma importante propriedade do BP é que, mesmo no pior caso, onde temos um custo exponencial (mas na prática, polinomial) ele encontrará todas as soluções (realizações) de uma instância dada.

No $\operatorname{EDMCP}^{3}$ (Problema de Completamento de Matrizes de Distâncias Euclidianas [3]), é dada uma matriz simétrica parcial $A$ (isto é, certas entradas de $A$ são desconhecidas) e desejamos obter como solução um par $(\bar{A}, K)$, onde $\bar{A}$ é um completamento simétrico de $A$ e $K \in \mathbb{N}$, tais que:

(a) $\bar{A}$ é uma matriz de distâncias euclidianas em $\mathbb{R}^{K} \mathrm{e}$

(b) $K$ é o mínimo possível.

Uma variante do $\mathrm{EDMCP}$ é o $\mathrm{EDMCP}_{K}$, onde $K$ é um dado inicial e desejamos obter (se possível) os completamentos possíveis $\bar{A}$ satisfazendo a condição (a) acima.

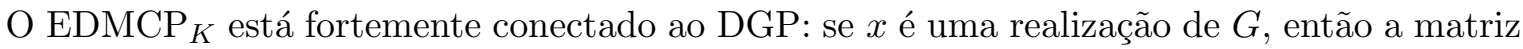
parcial $A$ pode ser completada em tempo polinomial e se $\bar{A}$ é um completamento válido de $A$, então o grafo correspondente é uma clique e sua realização em $\mathbb{R}^{K}$ pode ser encontrada em tempo polinomial [4]. Assim, obtemos uma correspondência entre as soluções do DGP e as soluções do $\mathrm{EDMCP}_{K}$ associado.

Cada instância $G=(V, E, d)$ do ${ }^{2}$ DMDGP pode ser transformada em tempo linear em uma instância de um $\mathrm{EDMCP}_{2}$ (e vice versa [4]). Considerando a ordem dada, definimos a forma padrão da matriz parcial de distâncias euclidianas $D$, do $\mathrm{EDMCP}_{2}$ associado, como sendo a matriz parcial $D=\left[d_{i j}\right]$, tal que $d_{i j}$ é a distância do vértice $i$ ao vértice $j$. Tal forma padrão garante que todos os elementos do conjunto $\left\{d_{i j}:|i-j| \leq 3\right\}$ são conhecidos.

Em [3], os autores exploram a possibilidade de um BP dual. Eles obervam que grafos ponderados e matrizes parciais simétricas são "duais" um do outro. Enquanto no primal procuramos por realizações do grafo, no dual procuramos por completamentos de matrizes parciais de forma que realizações e completamentos de matrizes de distâncias também são duais um do outro.

${ }^{3}$ Do inglês: Euclidean Distance Matrix Completion Problem 
Enquanto o BP (primal) decide sobre pontos $x_{v} \in \mathbb{R}^{K}$ que realizam o vértice $v$, o BP dual decide sobre distâncias desconhecidas $\delta$ associadas a $v$.

Vamos denotar por $\mathrm{CM}(D)$ o determinante de Cayley-Menger [2] associado à matriz de distâncias euclideanas $D$. Os dois resultados abaixo (veja [2]) serão úteis mais adiante:

Proposição 1.1. Considere a matriz parcial de distâncias $D=\left[d_{i j}\right]$ abaixo, tal que a entrada $\delta=d_{14}^{2}$ é desconhecida:

$$
D=\left[\begin{array}{cccc}
0 & d_{12}^{2} & d_{13}^{2} & \delta \\
d_{21}^{2} & 0 & d_{23}^{2} & d_{24}^{2} \\
d_{31}^{2} & d_{32}^{2} & 0 & d_{34}^{2} \\
\delta & d_{42}^{2} & d_{43}^{2} & 0
\end{array}\right]
$$

A equação quadrática $\operatorname{CM}(D)=0$ (os valores de $\delta$ que anulam $\operatorname{CM}(D)$ ) tem solução real se, e somente se, $D$ é uma matriz de distâncias euclidianas. Neste caso, com probabilidade 1, ela tem duas soluções distintas $\delta^{1}, \delta^{2}$ que são dois valores válidos para a distância desconhecida $d_{14}^{2}$.

Proposição 1.2. Considere uma matriz parcial $D=\left[d_{i j}\right]$ de distâncias, tal que apenas a distância $d_{1 n}$ é desconhecida, com $n \geq 5$. Façamos $\delta=d_{1 n}^{2}=\left\|v_{1}-v_{n}\right\|^{2}$. Então, a equação quadrática $\operatorname{CM}(D, \delta)=0$ tem uma única solução real, que é o único valor válido para a distância desconhecida $d_{1 n}$.

\section{Número de Soluções: uma Interpretação Dual}

Nesta seção, apresentaremos um resultado (em $[1,5])$ sobre o número de realizações válidas de uma instância $G=(V, E, d)$ de um ${ }^{2}$ DMDGP, usando apenas o "espaço de distâncias", ou seja, a contagem será feita a partir das matrizes parciais do $\mathrm{EDMCP}_{2}$ associado. Se $X=$ $\left\{x_{1}, x_{2}, \ldots, x_{N}\right\}$ é o conjunto de todas as realizações válidas, iremos determinar a cardinalidade de $X$ a partir da matriz parcial $D$ do $\mathrm{EDMCP}_{2}$ associado.

Seja $D=\left[d_{i j}\right]$ a matriz parcial do $\mathrm{EDMCP}_{2}$ associado a um ${ }^{2}$ DMDGP dado. Devido à simetria, consideramos apenas $i \leq j$ para $i, j=1, \cdots, n$.

Definição 2.1. Definimos a $k$-ésima diagonal (ou simplesmente $k$-diagonal) como a subdiagonal de $D$ cujos elementos satisfazem $j-i=k$, para $k=0, \cdots, n-1$. Chamaremos de $(i, j)$-submatriz principal de $D$ à submatriz $\hat{D}_{i, j}$, de ordem $j-i+1$, dada por $\left(d_{r s}\right)_{r, s=i}^{j}$, ou seja, constituída pelas linhas $i \leq r \leq j$ e colunas $i \leq s \leq j$ de $D$.

Para determinar o número de completamentos para a matriz parcial $D$, vamos inicialmente considerar o exemplo abaixo.

Exemplo 2.2. Considere a seguinte matriz parcial de distâncias, associada a uma instância com 7 vértices:

$$
D=\left[\begin{array}{ccccccc}
0 & \bullet & \bullet & ? & ? & ? & ? \\
& 0 & \bullet & \bullet & ? & \bullet & ? \\
& & 0 & \bullet & \bullet & ? & ? \\
& & & 0 & \bullet & \bullet & ? \\
& & & & 0 & \bullet & \bullet \\
& & & & & 0 & \bullet \\
& & & & & & 0
\end{array}\right]
$$

Observe que conhecemos a entrada $(2,6)$ da matriz $D$. Logo, só teremos uma possibilidade para as entradas $(2,5),(3,6)$. De fato, de acordo com a Proposição 1.2 aplicada à $(2,6)$-submatriz principal, cada par de possibilidades para as entradas $(2,5),(3,6)$ geraria um valor para entrada $(2,6)$, e tais valores são distintos com probabilidade um. Assim, só temos uma possibilidade para o par $\{(2,5),(3,6)\}$. 
De acordo com a Proposição 1.1, obtemos ainda (via equação de Cayley-Menger) duas possibilidades para as entradas $(1,4)$ e $(4,7)$ na 3-diagonal. Aplicando a Proposição 1.2 às submatrizes principais associadas as entradas que restam, obtemos via princípio multiplicativo, duas possibilidades para cada entrada restante:

$$
B=\left[\begin{array}{lllllll}
0 & \bullet & \bullet & 2 & 2 & 2 & 4 \\
& 0 & \bullet & \bullet & 1 & \bullet & 2 \\
& & 0 & \bullet & \bullet & 1 & 2 \\
& & & 0 & \bullet & \bullet & 2 \\
& & & & 0 & \bullet & \bullet \\
& & & & & 0 & \bullet \\
& & & & & & 0
\end{array}\right]
$$
$\mathbb{R}^{2}$.

Portanto, temos 4 completamentos distintos para $D$ e, consequentemente, 8 realizações em

Teorema 2.3. Dada uma instância de um ${ }^{2}$ DMDGP $G=(V, E)$, com $|V|=n$, considere as distâncias $d_{i j}$ da instância do $\mathrm{EDMCP}_{2}$ associada, dada de acordo com a ordem em $V$ que garante que todas as distâncias $d_{i j}$, com $|i-j| \leq 2$, são conhecidas (a matriz $D=\left[d_{i j}\right]$ está na forma padrão). Considere ainda, fixadas as posições dos três primeiros vértices $v_{1}, v_{2}, v_{3}$. Assim, com probabilidade um, temos que:

1. Se não existe informação de poda, então existem apenas 2 possíveis valores reais distintos para as distâncias $d_{i, i+3}, i=1, \ldots, n-3$, na 3 -diagonal da matriz $D=\left[d_{i j}\right]$.

2. Se todas as distâncias $d_{i, i+3}, i=1, \ldots, n-3$, na 3 -diagonal são conhecidas, então existe uma única solução para o $\mathrm{EDMCP}_{2}$.

3. O número de possibilidades distintas para a distância $d_{1 n}$ é igual ao produto do número de possibilidades para cada elemento da 3 -diagonal $d_{i, i+3}, i=1, \ldots, n-3$.

4. Se a distância $d_{1 n}$ é conhecida, então existe apenas uma solução para o $\mathrm{EDMCP}_{2}$.

O resultado acima é mais geral (válido para $K \geq 1$ ) e pode ser encontrado em $[1,5]$.

\section{Um pouco sobre Bilateração}

Vamos denotar por $p_{i}$ o vetor posição de um ponto $P_{i}$ (em algum espaço euclidiano) e por $p_{i, j}$ o vetor $\overrightarrow{P_{i} P_{j}}$. Seja

$$
\mathrm{CM}\left(i_{1}, \ldots, i_{n} ; j_{1}, \ldots, j_{n}\right)=2\left(\frac{-1}{2}\right)^{n}\left|\begin{array}{cccc}
0 & 1 & \cdots & 1 \\
1 & d_{i_{1}, j_{1}}^{2} & \cdots & d_{i_{1}, j_{n}}^{2} \\
\vdots & \vdots & \ddots & \vdots \\
1 & d_{i_{n}, j_{1}}^{2} & \cdots & d_{i_{n}, j_{n}}^{2}
\end{array}\right|,
$$

o bideterminante de Cayley-Menger das sequências de pontos $P_{i_{1}}, \ldots, P_{i_{n}}$ e $P_{j_{1}}, \ldots, P_{j_{n}}$, onde $d_{i_{k}, j_{\ell}}=\left\|P_{j_{\ell}}-P_{i_{k}}\right\|$. Quando as sequências de pontos são as mesmas, $C M\left(i_{1}, \ldots, i_{n}\right)$ denota o determinante de Cayley-Menger da sequência de pontos $P_{i_{1}}, \ldots, P_{i_{n}}$ [7].

Os determinantes de Cayley-Menger podem ser usados para modelar um grande número de problemas geométricos [2]. O valor de $\operatorname{CM}\left(i_{1}, \ldots, i_{n}\right)$ é igual a $(n-1) !^{2}$ vezes o quadrado do hiper-volume do simplex gerado pelos pontos $P_{i_{1}}, \ldots, P_{i_{n}}$ em $\mathbb{R}^{n-1}$ [2]. Particularmente, a área orientada do triângulo determinado por $P_{i}, P_{j}, P_{k}$ é igual a $\pm \frac{1}{2} \sqrt{\mathrm{CM}(i, j, k)}$ (o sinal depende da posiçao de $P_{k}$ em relação à reta definida por $P_{i}$ e $\left.P_{j}\right)$. Também é fato que $\operatorname{CM}\left(i_{1}, i_{2} ; j_{1}, j_{2}\right)$ é equivalente ao produto interno entre os vetores $p_{i_{1}, i_{2}}$ e $p_{j_{1}, j_{2}}$.

A bilateração consiste em encontrar a posição relativa de um ponto $P_{k}$, dadas as suas distâncias a dois outros pontos $P_{i}$ e $P_{j}$ cujas posições são conhecidas. De acordo com [7], temos que: 


$$
p_{i, k}=\frac{\mathrm{CM}(i, j: i, k)}{\operatorname{CM}(i, j)} p_{i, j} \pm \frac{\sqrt{\mathrm{CM}(i, j, k)}}{\operatorname{CM}(i, j)} S p_{i, j}=\frac{1}{\operatorname{CM}(i, j)}(\operatorname{CM}(i, j ; i, k) \pm \sqrt{\operatorname{CM}(i, j, k)} S) p_{i, j},
$$

onde $S=\left(\begin{array}{cc}0 & -1 \\ 1 & 0\end{array}\right)$. A equação (4) pode ser reescrita como:

$$
p_{i, k}=Z_{i, j, k} p_{i, j}
$$

onde

$$
Z_{i, j, k}=\frac{1}{\operatorname{CM}(i, j)}\left(\begin{array}{cc}
\mathrm{CM}(i, j ; i, k) & \mp \sqrt{\operatorname{CM}(i, j, k)} \\
\pm \sqrt{\operatorname{CM}(i, j, k)} & \operatorname{CM}(i, j ; i, k)
\end{array}\right)
$$

é dita matriz de bilateração. Tais matrizes têm propriedades interessantes (para mais detalhes, consulte [7]) e iremos destacar uma delas: $I-Z_{i, j, k}=Z_{j, i, k}$. Elas são matrizes perpendiculares, isto é, as linhas ou colunas são vetores ortogonais de mesma norma. Para matrizes perpendiculares $A, B$ valem as propriedades:

1. $A+B$ e $A B$ são perpendiculaares. Além disto, $A B=B A$;

2. $A A^{T}=A^{T} A=\operatorname{det}(A) I$, onde $A^{T}$ é a transposta de $A$ e $I$ a matriz identidade;

3. se $v=A w$ então $\|v\|^{2}=\operatorname{det}(A)\|w\|^{2}$.

\subsection{Dois triângulos com uma aresta em comum}

Vamos considerar agora dois triângulos com uma aresta em comum, como na figura abaixo:

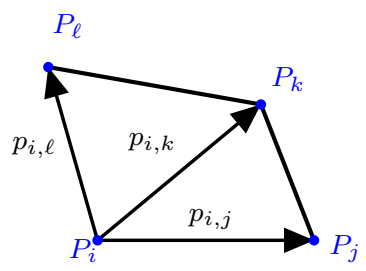

Observe que, aplicando duas bilaterações consecutivas, podemos obter $p_{i, \ell}$ em termos de $p_{i, j}$ :

$$
p_{i, \ell}=Z_{i, k, \ell} p_{i, k}=Z_{i, k, \ell} Z_{i, j, k} p_{i, j}
$$

De fato, qualquer vetor definido por dois pontos no conjunto $\left\{P_{i}, P_{j}, P_{k}, P_{\ell}\right\}$ pode ser expresso em função de $p_{i, j}$ por matrizes de bilateração. Por exemplo:

$$
p_{j, \ell}=p_{i, \ell}-p_{i, j}=\left(Z_{i, k, \ell} Z_{i, j, k}-I\right) p_{i, j} \Rightarrow d_{j, \ell}^{2}=\operatorname{det}\left(Z_{i, k, \ell} Z_{i, j, k}-I\right) d_{i, j}^{2} .
$$

Podemos generalizar este procedimento para uma "faixa" de triângulos que compartilham uma aresta. Este é o caso típico de um grafo que representa um ${ }^{2}$ DMDGP.

\subsection{Bilaterações sucessivas aplicadas ao ${ }^{2}$ DMDGP}

Vamos considerar instâncias com $5 \leq n$ vértices, onde a única informação de poda é $d_{1, n}$, isto é, a distância do primeiro ao último vértice. Pelo teorema sobre o número de soluções, existe apenas um completamento possível para a matriz incompleta $D=\left[d_{i j}\right]$. Por enquanto, vamos desconsiderar o ramo de soluções simétricas na árvore de busca, isto é, fixamos os vértices $v_{1}, v_{2}, v_{3}$. No nível $k(k \geq 4)$, o $\mathbf{B P}$ encontra duas possíveis posições para o vértice $v_{k}$, que geram 
dois valores distintos para distância desconhecida $d_{k-3, k}$. Só ocorrerá poda no último nível, após $2^{n-3}$ ramificações na árvore de busca.

Nosso objetivo é incorporar a bilateração ao BP para obter podas em todos os níveis, isto é, no nível $k$ temos uma "parte" da única (a menos da simetria) solução do problema. No que se segue, escreveremos triângulo $i j k$ para indicar o triângulo com vértices em $v_{i}, v_{j}, v_{k}$. Para exemplificar o procedimento que adotaremos, vamos considerar o caso $n=7$.

Inicialmente, o BP encontra posições para os três primeiros vértices e, no nível 4, encontra dois possíveis valores para a distância desconhecida $d_{14}$. Fazendo

$$
\begin{gathered}
p_{17}=p_{14}+p_{45}+p_{56}+p_{67}=p_{14}+\left(Z_{435} Z_{413}\right) p_{41}+\left(Z_{546} Z_{435} Z_{413}\right) p_{41}+\left(Z_{657} Z_{546} Z_{435} Z_{413}\right) p_{41}= \\
=\left(I-Z_{435} Z_{413}+Z_{546} Z_{435} Z_{413}-Z_{657} Z_{546} Z_{435} Z_{413}\right) p_{14},
\end{gathered}
$$

temos que

$$
d_{17}^{2}=\operatorname{det}\left(I+\left(-I+\left(I-Z_{657}\right) Z_{546}\right) Z_{435} Z_{413}\right) d_{14}^{2} .
$$

Esta última equação depende apenas das distâncias conhecidas e de $d_{14}$. Pelo Teorema (2.3) acima, apenas o valor correto para $d_{14}$ satisfaz (8). Com apenas uma substituição, encontramos o valor correto de $d_{14}$ e efetuamos a poda. Enfatizamos que na definição da matriz $Z_{i, j, k}$ em (6) temos duas possibilidades de sinal mas no cálculo do determinante em (8) temos 8 possibilidades (distintas) apenas: basta considerar a quantidade de sinais negativos no produto das matrizes de bilateração.

No próximo nível, o BP calcula dois pares de possibilidades $\left(d_{25}, d_{15}\right)$ (para cada valor de $d_{25}$ corresponde um único valor de $d_{15}$ ). Repetimos o procedimento anterior e obtemos

$$
d_{17}^{2}=\operatorname{det}\left(I+\left(-I+Z_{657}\right) Z_{546} Z_{514}\right) d_{15}^{2} .
$$

Apenas um dentre os dois valores possíveis para $d_{15}$ satisfaz esta equação. Assim, com apenas uma substituição, encontramos o par correto $\left(d_{25}, d_{15}\right)$ e efetuamos a poda.

No nível 6 , o BP calcula duas ternas de possibilidades $\left(d_{36}, d_{26}, d_{16}\right)$ e, um valor correto determina unicamente os outros dois. Aplicando a bilateração, obtemos

$$
d_{17}^{2}=\operatorname{det}\left(I-Z_{617}\right) d_{16}^{2},
$$

e apenas um dentre os dois valores possíveis para $d_{16}$ satisfaz esta equação. Assim, com apenas uma substituição, encontramos a terna correta $\left(d_{36}, d_{26}, d_{16}\right)$ e efetuamos a poda.

No próximo nível, o BP encontra a solução.

\subsubsection{Caso Geral:}

Para $n \geq 5$ arbitrário, o $\mathbf{B P}$ determina posições para os três primeiros vértices e no nível $k$ $(4 \leq k \leq n-1)$ calcula possibilidades para as distâncias que faltam $\left\{d_{i k}: 1 \leq i \leq k-3\right\}$ e efetuamos a poda através de bilaterações sucessivas, como segue abaixo:

$$
d_{1 n}^{2}=\operatorname{det}\left(I+\left(-I+\left(\cdots+\left((-1)^{n-k+1}\left(I-Z_{n-1, n-2, n}\right)\right) \cdots\right) Z_{k+1, k, k+2}\right) Z_{k, k-1, k+1} Z_{k, 1, k-1}\right) d_{1 k}^{2} .
$$

No nível $k$, a equação acima envolve apenas distâncias conhecidas (e aquelas obtidas nos níveis anteriores) e a incógnita $d_{1 k}$. Temos sempre duas possibilidades para $d_{1 k}$, e após o teste na equação (9) encontramos o valor correto para esta distância. Neste nível, o BP calculou duas $(k-3)$-uplas de possibilidades para a coluna $k:\left(d_{1 k}, \ldots, d_{k-3, k}\right)$. Desta forma, os demais valores desconhecidos na coluna $k$ de $D$ ficam unicamente determinados (Teorema 2.3) a partir do valor correto de $d_{1 k}$. 


\section{Conclusões}

Dada uma matriz parcial de distâncias euclidianas $D=\left[d_{i j}\right]$ associada a um ${ }^{2}$ DMDGP, podemos usar a bilateração para reduzir a árvore de busca do BP. Se uma certa distância $d_{i j},|i-j| \geq 5$ é uma informação de poda, tal que não existem informações adicionais nas colunas intermediárias $i<k<j$, então a partir do nível $i+4$ aplicamos a equação (9) para " antecipar" a poda que só ocorreria no nível $j$. Desta forma, ocorrem podas em todos os níveis intermediários $i<k<j$ e, no nível $j$, temos apenas soluções factíveis.

O custo computacional é o mesmo do BP em virtude dos produtos de matrizes envolvidos, mas não armazenamos soluções infactíveis a cada nível do BP. A bilateração também pode ser usada para determinar a distância $d_{i j}$, quando conhecemos toda a 3-diagonal entre $i$ e $j$, sem fazer uso de coordenadas. Num futuro próximo, pretendemos aplicar a trilateração ao problema em $\mathbb{R}^{3}$ para obter resultados semelhantes.

\section{Agradecimentos}

Este trabalho contou com o apoio da FAPESP, FAPEMIG, CNPq e CAPES.

\section{Referências}

[1] G. Abud; J. Alencar, Counting the number of solutions of the Discretizable Molecular Distance Geometry Problem. Workshop on Distance Geometry and Applications - DGA, 2013, Manaus.

[2] L. Blumenthal, Theory and Applications of Distance Geometry. Oxford University Press, Oxford, 1953

[3] L. Liberti; C. Lavor, On a relationship between graph realizability and distance matrix completion. Optimization Theory, Decision Making, and Operations Research Applications. Springer, p. 39-48, 2013.

[4] L. Liberti; C. Lavor; N. Maculan; A. Mucherino, Euclidean distance geometry and applications, SIAM Review, v. 56, p. 3-69, 2014.

[5] L. Liberti; C. Lavor; J. Alencar; G. Abud, Counting the number of solutions of K-DMDGP instances. Lecture Notes in Computer Science, v. 8085, p. 224-230, 2013.

[6] K. Menger, Untersuchungen über allgemeine Metrik, Mathematische Annalen, v. 100, p. 75-163, 1928.

[7] N. Rojas; F. Thomas, The Forward Kinematics of 3-R $\underline{P}$ R Planar Robots: A Review and a Distance-Based Formulation, IEEE Transactions on Robotics 27, p. 143-150, 2011. 